\title{
GRIPS and the Perspective of Next-generation Gamma-ray Surveys
}

\author{
Roland Diehl* \\ and the GRIPS collaboration ${ }^{\dagger}$ \\ Max Planck Institut für extraterrestrische Physik, D-85748 Garching, Germany \\ E-mail: rod@mpe.mpg.de
}

\begin{abstract}
GRIPS is one example of next generation telescopes proposed for astronomy the energy range between hard X-ray mirror instruments such as NuStar and the Fermi telescope. The Compton telescope principle is an advantageous concept in view of background suppression, imaging sensitivity within a large field of view and energy range, and capability to measure polarization. The diversity of astrophysical sources at high energies (diffuse emission from cosmic-ray interactions, nuclear lines from point-like and diffuse sources, accreting binaries, cosmic-ray acceleration sites, novae and supernovae, GRBs) presents a challenge, and in particular emphasizes the need for large fields of view and surveys. We discuss the astrophysical challenges which are expected to remain after the extended INTEGRAL mission, and how such a next-generation survey at low-energy gamma-rays would impact on these. We argue that qualitatively new and more direct insights could be obtained on cosmic high-energy phenomena and their underlying physical processes.
\end{abstract}

$8^{\text {th }}$ INTEGRAL Science Workshop

The Restless Gamma-Ray Universe

October 27-30, 2010

Dublin, Ireland

\footnotetext{
*Speaker.

${ }^{\dagger}$ www.grips-mission.eu
} 


\section{Science Issues in High-Energy Astrophysics}

The field of high-energy astrophysics is very broad, both in its observational windows and in its astrophysical diversity. Often, high energies are understood as high temperatures, meaning the hottest cosmic objects radiating in thermal emission, i.e. cosmic plasma radiating at X-ray energies. Candidate sources are accreting compact objects, from white dwarfs through neutron stars and black holes from stellar to supermassive, and gamma-ray bursters. One may, alternatively, associate high-energy source processes with the field of high-energy astrophysics, which then encompasses non-thermal processes such as radioactive decay and nuclear de-excitation, Bremsstrahlung, synchrotron and inverse-Compton radiation, and pion decay. In this case, the energy bands of interest cover about 8-10 orders of magnitude on the frequency scale, and hence a diversity of groundand space-based telescopes. The astrophysical issues discussed in the context of high-energy astrophysics comprise the origins of cosmic rays and relativistic particle acceleration, the physics in extreme gravitational or magnetic fields (including accretion onto compact objects, and pulsars and magnetars, the astrophysics of cosmic explosions (supernovae, novae, gamma-ray bursts, and type-I X-ray bursters), and cosmic nucleosynthesis sources.

These astrophysical question remain in the focus of science:

- Which are the Cosmic Sources of non-thermal Emission?

- How did Massive Stars influence the Early Universe and Galaxies?

- What is the Role of SMBHs in Galaxies (Blazars)?

- How does Matter flow onto Compact Objects, and make Jets?

- How do Supernova Explosions work?

- How are Cosmic-Ray Particles accelerated?

- How do Pulsars and Magnetars work?

- What can we learn from the Sun on Particle Acceleration?

- How do Positrons propagate and annihilate?

- Is there a HE Signature from Dark Matter?

Considering present and planned astronomical facilities for high-energy astrophysics, we can see that the study of following cosmic objects is well underway: Accreting compact objects, cosmic relativistic particle acceleration in supernova remnants, accreting binaries, pulsars, and active galaxies. Less well covered still are the less-efficient radiation from more diluted cosmic plasma in hot interstellar and intergalactic medium, and of radiation signatures related to atomic nuclei.

\section{High-Energy Astrophysics Experiments}

INTEGRAL [2] contributed in its way to this field through data at hard-X and low-energy gamma-rays. Major astrophysical findings were the deeply-embedded sources (mostly high-mass $\mathrm{X}$-ray binaries, i.e. young and massive stars), the high-energy emission tails from extremelymagnetized neutron stars, and the puzzling morphology of positron annihilation emission throughout the Galaxy. New insights on what might be useful astronomical challenges for astrophysics insights became evident from the polarization signatures in the Crab and a gamma-ray burst (GRB041219a), from the new source types such as embedded high-mass binaries, the magnetized neutron stars, and the binaries interacting with their surroundings, all of which only became visible at energies 
above the $10-\mathrm{keV}$ regime. More insights into nuclear emission was obtained from the spectroscopic precision in the bright ${ }^{26} \mathrm{Al}$ line and from detection of ${ }^{60} \mathrm{Fe}$, but the hoped-for detections of the plausibly-expected nuclear emission in the $\mathrm{MeV}$ range from high-energy collisions could not be obtained. Thus, gamma-ray line spectroscopy could provide interesting and new insights on massive-star interiors through the ${ }^{26} \mathrm{Al}$ and ${ }^{60} \mathrm{Fe}$ measurements. But neither the promises from nova lines $\left({ }^{9} \mathrm{Be}\right.$ and $\left.{ }^{22} \mathrm{Na}\right)$ nor from supernova lines $\left({ }^{56,57} \mathrm{Ni}\right.$ and $\left.{ }^{44} \mathrm{Ti}\right)$ with their potential to clarify these cosmic explosions from measurements related to their interiors could really be fulfilled. Nuclear de-excitation lines (at 2.2 MeV from neutron captures, and 4.4 or $6.1 \mathrm{MeV}$ from ${ }^{12} \mathrm{C}$ and ${ }^{16} \mathrm{O}$ de-excitation were seen, expected to be smoking guns of low-energy cosmic ray interactions in interstellar space or near compact objects. INTEGRAL was not sensitive enough to see at least a small sample of such sources.

ESA's Cosmic Vision program provides a framework to gather ideas on promising space-based experiments for further insights, with above high-energy astrophysics themes included explicitly in its scope and list of themes: Theme 3 of Cosmic Vision is called the evolving high-energy universe, and includes the themes discussed in the previous Section, although $\gamma$-rays are explicitly mentioned only in the context of black holes, surprisingly.

\section{The GRIPS Perspective}

Among the diversity of concepts pursued to measure cosmic photons at energies above the regime of focusing optics (i.e. X-rays up to possibly $100 \mathrm{keV}$ ) and below the regime of groundbased Cerenkov or air shower instruments, the tracking chamber concept appears most promising in collecting photon interaction events both efficiently and also including imaging and backgroundrejection information in the measurement. At the low-energy end, interactions of photons with detector material occur mostly through Compton scattering (up to several MeV), while towards higher energies pair creation becomes the dominant interaction process. The main instrument for the GRIPS mission proposed for ESA's Cosmic Vision program and described below [1], therefore, is a Compton telescope with a Si tracker as its central component, to provide secondary-particle identification and tracking for both these photon interaction types.

The MeV-gap in current instrument sensitivity encompasses the photon energy range between hard X-rays of $0.2 \mathrm{MeV}$ and $\gamma$-rays of $80 \mathrm{MeV}$ (Fig. 1). The GRIPS mission [?] is designed to improve the sensitivity here by a factor of 40 compared to previous missions. Therefore, the GRIPS all-sky survey with $\gamma$-ray imaging, polarimetry, and spectroscopy holds a high promise of new discoveries and of precision diagnostics of primary high-energy processes.

\subsection{Instrumentation and Mission}

The GRIPS concept combines a Compton and pair telescope based on the latest developments in nuclear and high-energy physics laboratories (Fig. ??). Modern 3D position-sensitive and spaceproven detectors with advanced (nanosecond level) readout technology will ensure unprecedented background rejection capability to guarantee the above sensitivity leap. Taking advantage of the Compton-scattering physics, GRIPS will also be a very sensitive polarimeter. The energy resolution of $3 \%$ at photon energies of $1 \mathrm{MeV}$ renders the gamma-ray telescope ideal for the study of broadened emission lines from cosmic explosions such as supernovae. Moreover, the extent of 


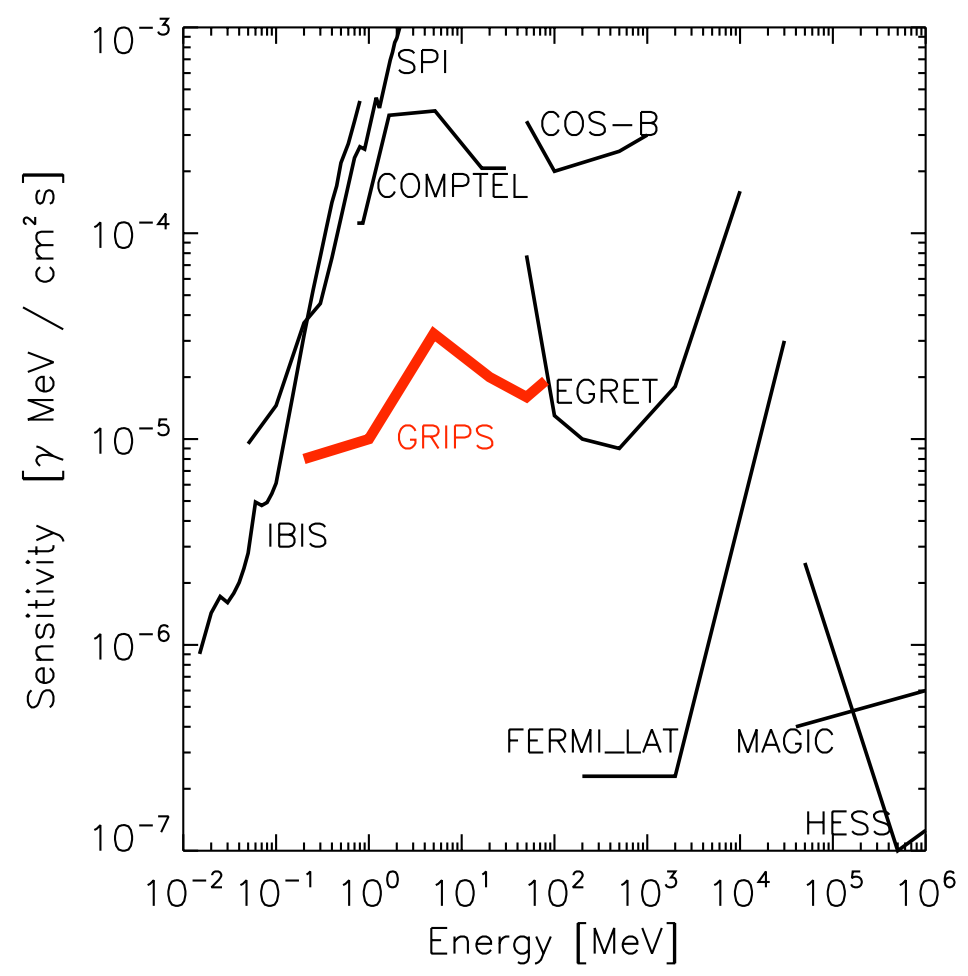

Figure 1: GRIPS will allow a major sensitivity improvement in an energy range (between hard X-rays and $\mathrm{GeV} \gamma$-rays) which has been poorly explored, yet holds unique information for a wide range of astrophysical questions. The curves are for an exposure of $10^{6} \mathrm{sec}, \Delta E=E$, and an $E^{-2}$ spectrum.

such spectroscopic performance throughout the entire nuclear energy range should finally enable pioneering astrophysical studies of nuclear de-excitation and nuclear resonance absorption lines, and thus enable nuclear line spectroscopy similar to the rich field of atomic line spectroscopy, thus expanding beyond the radioactivity lines studied up to now. The limitation in imaging resolution which is intrinsic to the detection physics in the $\mathrm{MeV}$ band can be compensated by detecting secondary emission from the same sources with the foreseen auxiliary X-ray and NIR telescopes with their sub-arcmin angular resolutions.

GRIPS should be launched a Soyuz-Fregate rocket launched from Kourou for a projected lifetime of 10 years into a low-altitude, equatorial orbit (LEO) to minimize the background. GRIPS consists of two satellites, flying in a close-pair configuration (Fig. 2): one satellite with the main instrument, the Gamma-Ray Monitor (GRM) including $500 \mathrm{~kg}$ of $\mathrm{LaBr}_{3}$ scintillator crystals, the other with auxilliary instruments, an X-Ray Monitor XRM and an Infra-Red Telescope (IRT). Both satellites should be 3-axis stabilized. The gamma-ray telescope/satellite of GRIPS is planned to be continuously pointing at the zenith, thus monitoring ca. $80 \%$ of the sky over each orbit for transient events, including gamma-ray bursts. The X-ray/infrared telescope satellite should have the capability of autonomously slewing for follow-up observations of gamma-ray bursts. As an added value, positions and fluxes of transient alerts can be provided to the community. Gamma-ray bursts in the redshift range $7<z<35$ would be recognized within minutes by IRT, and report in 
near-real-time.
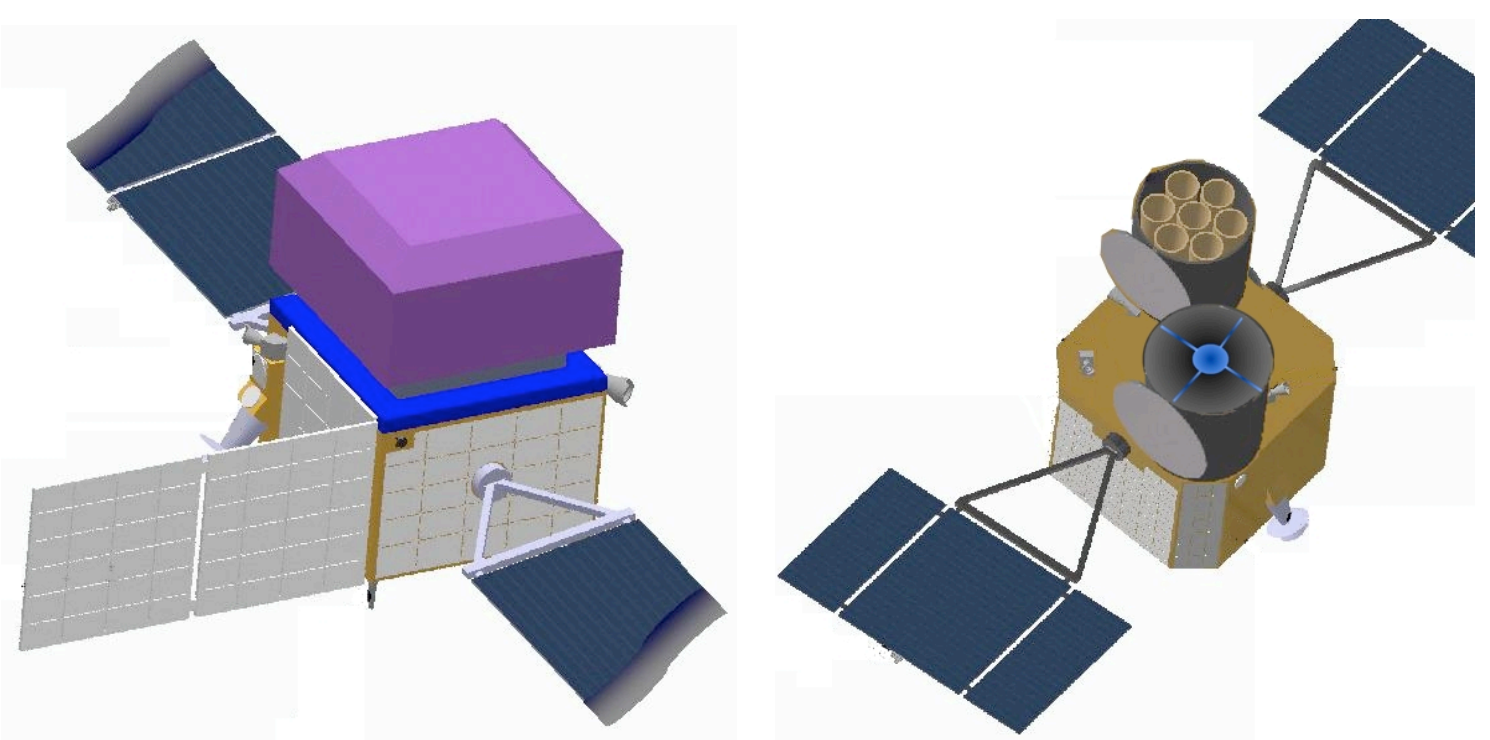

Figure 2: GRIPS configuration in the two-satellite option, where the GRM is on one satellite (left), and XRM and IRT on the other (right). The GRM satellite would just do the zenith scanning all-sky survey, while XRM/IRT would re-point with the whole (second) satellite to GRBs, similar to Swift.

\subsection{Prospected Science Results}

Gamma-Ray Bursts and First Stars: Unrivaled by any other method, the detection of highly penetrating $\gamma$-rays from cosmological $\gamma$-ray bursts shed light on the first massive stars and galaxies which formed during the dark ages of the early Universe. With its energy coverage up to $80 \mathrm{MeV}$, GRIPS could firmly establish the high energy component seen in addition to the canonical Band funtion in one CGRO/EGRET ( $>10 \mathrm{MeV}$ ) and one Fermi/LAT burst $(>100 \mathrm{MeV})$ in much larger numbers, and characterize its origin through polarisation signatures. GRIPS could measure the degree of polarisation of the prompt $\gamma$-ray burst emission to a few percent accuracy for more than $10 \%$ of the detected GRBs, and securely measure how the degree of polarisation varies with energy and/or time over the full burst duration for dozens of bright GRBs. Also, the delay of GeV photons relative to emission at $\sim$ hundred $\mathrm{keV}$, observed in a few GRBs with Fermi/LAT, manifests itself already at $\mathrm{MeV}$ energies in Fermi/GBM, and would thus be a science target for GRIPS. These observations could thus enable a clear identification of the prompt GRB emission processes, and determine the role played by magnetic fields.

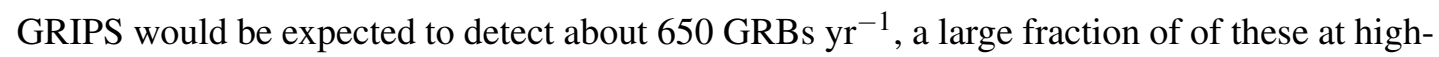
redshift ( $\sim 30 \mathrm{GRBs} \mathrm{yr}^{-1}$ at $z>5$, and $\sim 22$ GRBs at $z>10$ ). The 7-channel near-infrared telescope (IRT) would improve the localization to the required arcsecond level and determine photometric redshifts for the bulk of the most distance $(z>7)$ sources. It would allow to measure the incidence of gas and metals through X-ray absorption spectroscopy and line-of-sight properties by enabling NIR spectroscopy with JWST. 
If the GRB environments contain total hydrogen column densities of $10^{25} \mathrm{~cm}^{-2}$, or higher, GRIPS holds the promise of measuring redshifts directly from the $\gamma$-ray spectrum via nuclear resonances, and will be sensitive to do so beyond $\mathrm{z} \sim 13$.

GRIPS would also detect a handful of short GRBs at $z<0.1$, enabling a potential discovery of correlated gravitational-wave and/or neutrino signal.

Blazars: GRIPS would catalogue about 2000 blazars, probing blazar evolution to large redshifts. Such observations pinpoint the most massive halos at large redshifts, thus could severely constrain models of structure evolution. Such a large sample could establish their (evolving) luminosity function and thus determine the fractional contribution of blazars to the diffuse extragalactic background. GRIPS is expected to detect $\sim 10$ blazars at $\mathrm{z}>8$. Studies of the nonthermal radiation mechanisms would be supported through spectro-polarimetric measurements. The link between the inner accretion disk and the jet could be probed with correlated variability from the thermal to the nonthermal regime, using GRIPS auxiliary instruments. This would localize the region of high-energy emission.

Supernovae and Nucleosynthesis: The primary energy source of supernova (SN) light is radioactive decay. The first direct measurement of the Ni decay chain gamma-rays in Type Ia $\mathrm{SNe}$ would provide key insight into their explosion physics and disentangle progenitor channels. This would place the luminosity calibration of Type Ia SNe on physical rather than empirical footings, for these events that are considered as standard candles in cosmology. The otherwise unobtainable direct measurement of the inner ejecta and the explosive nucleosynthesis of core collapse supernovae through ${ }^{56} \mathrm{Ni} /{ }^{44} \mathrm{Ti}$ would allow to establish a physical model for these important terminal stages of massive-star evolution. Explosion asymmetries and the links to long GRBs are important aspects herein. The fraction of nearby pair-instability supernovae from very massive stars would be unambiguously identified through their increased brightness from copious radioactivity. It is such observations which are crucial for complementing envisaged neutrino and gravitational wave measurements, and for our understanding of cosmic chemical evolution.

Cosmic Rays: Nuclear de-excitation lines of abundant isotopes like ${ }^{12} \mathrm{C}$ and ${ }^{16} \mathrm{O}$, the hadronic fingerprints of cosmic-ray acceleration, are expected to be discovered with GRIPS. Understanding the relative importance of leptonic and hadronic processes, and the role of cosmic rays in heating and ionizing molecular clouds will boost our understanding of both relativistic-particle acceleration and the cycle of matter.

Magnetars: The measurements of instabilities in the magnetospheres of magnetars, which are expected to lead to few-hundred $\mathrm{keV}$ to possibly $\mathrm{MeV}$-peaked emission, could provide new aspects for the field of plasma physics and magnetic field impacts therein. Note that solar flare origins are still unclear, and the extreme magnetar flares may shed light on reconnections or other readjustments in magnetic fields far from the surface of a star.

Annihilation of Positrons: The riddle of positron annihilation in the Galaxy is in need for constraining the different candidate annihilation source types. GRIPS could search for positron escape from candidate sources along the galactic plane through annihilation $\gamma$-rays in their vicinity. For several microquasars and pulsars, point-source like appearance is expected if the local annihilation fraction $f_{\text {local }}$ exceeds $10 \%\left(\mathrm{I}_{\gamma} \sim 10^{-2} \cdot f_{\text {local }} \mathrm{ph} \mathrm{cm}^{-2} \mathrm{~s}^{-1}\right)$. With GRIPS we could crosscorrelate annihilation $\gamma$-ray images with candidate source distributions, such as ${ }^{26} \mathrm{Al}$ and Galactic diffuse emission above $\mathrm{MeV}$ energies (where it is dominated by cosmic-ray interactions with the 
ISM) [both also measured with GRIPS at superior quality], point sources derived from INTEGRAL, Swift, Fermi, and H.E.S.S./MAGIC/VERITAS/CTA measurements, and with candidate dark-matter related emission profiles. GRIPS wouldl deepen the current INTEGRAL sky sensitivity by at least an order of magnitude in flux, at similar angular resolution. Comparing Galactic-disk and -bulge emission, limits on dark-matter produced annihilation emission could be placed, and constrain decay channels from neutralino annihilation in the gravitational field of our Galaxy. GRIPS could also perform sensitive searches for $\gamma$-ray signatures of dark matter for nearby dwarf galaxies.

Solar Flares: Solar flare observations would be a natural by-product of the continuous sky survey carried out by GRIPS since the Sun passes regularly through its field of view. Gamma-rays in the $\mathrm{MeV}$ regime provide the means to directly probe particle acceleration and matter interactions in these magnetised, non-thermal plasmas. Polarisation measurements are of great value for disentangling these dynamic processes.

\section{Summary}

Astrophysics of high-energy processes needs to close the observational gap remaining from the relatively poor sensitivity obtained so far in the regime between hard $\mathrm{X}$-rays and $\mathrm{GeV}$ or higherenergy gamma-rays. This range of the electromagnetic spectrum of cosmic photons holds unique messages which cannot be obtained otherwise, and also allows to measure directly several key processes which currently are claimed to be understood or explored yet depend on sometimes uncertain models or assumed parameters. GRIPS is one of the ideas to address this, and to invest thus into qualitatively new astronomy.

Acknowledgements The GRIPS consortium has discussed the astrophysical issues to be addressed and has assembled all project-related design and planning material. We appreciate the constructive work of this consortium over the past years, including the preparation of mission idea proposals for ESA's Cosmic Vision scheme.

\section{References}

[1] J. Greiner, A. Iyudin, G. Kanbach, A. Zoglauer, R. Diehl, F. Ryde, D. Hartmann, A. V. Kienlin, S. McBreen, M. Ajello, Z. Bagoly, L. G. Balasz, G. Barbiellini, R. Bellazini, L. Bezrukov, D. V. Bisikalo, G. Bisnovaty-Kogan, S. Boggs, A. Bykov, A. M. Cherepashuk, A. Chernenko, W. Collmar, G. Dicocco, W. Dröge, M. Gierlik, L. Hanlon, I. Horvath, R. Hudec, J. Kiener, C. Labanti, N. Langer, S. Larsson, G. Lichti, V. M. Lipunov, B. K. Lubsandorgiev, A. Majczyna, K. Mannheim, R. Marcinkowski, M. Marisaldi, B. McBreen, A. Meszaros, E. Orlando, M. I. Panasyuk, M. Pearce, E. Pian, R. V. Poleschuk, A. Pollo, A. Pozanenko, S. Savaglio, B. Shustov, A. Strong, S. Svertilov, V. Tatischeff, J. Uvarov, D. A. Varshalovich, C. B. Wunderer, G. Wrochna, A. G. Zabrodskij, and L. M. Zeleny. Gamma-ray burst investigation via polarimetry and spectroscopy (GRIPS). Experimental Astronomy, pages 23-+, July 2008.

[2] C. Winkler, T. J.-L. Courvoisier, G. Di Cocco, N. Gehrels, A. Giménez, S. Grebenev, W. Hermsen, J. M. Mas-Hesse, F. Lebrun, N. Lund, G. G. C. Palumbo, J. Paul, J.-P. Roques, H. Schnopper, V. Schönfelder, R. Sunyaev, B. Teegarden, P. Ubertini, G. Vedrenne, and A. J. Dean. The INTEGRAL mission. A\&A, 411:L1-L6, November 2003. 